\title{
Apuntes (des)confinados. Sobre las contribuciones de la interseccionalidad a los desafíos del Covid- I 9
}

\section{(De)confined notes. On the contributions of intersectionality to the challenges of Covid- 19}

\author{
Paulina de los Reyes ${ }^{1}$ \\ Universidad de Estocolmo, Suecia.
}

Recibido: 07/04/2021

Aceptado: 02/06/2021

\section{Cómo citar}

de los Reyes, P. (2021). Apuntes (des)confinados. Sobre las contribuciones de la interseccionalidad a los desafíos del Covid-19. Propuestas Críticas en Trabajo Social - Critical Proposals in Social Work 1(2), 31-49. DOI: 10.5354/2735-6620.2021.61524

\section{Resumen}

Este artículo examina de qué manera las perspectivas interseccionales contribuyen a profundizar el análisis de las operaciones del poder, en un contexto de crisis sanitaria, entregando también una reflexión sobre las premisas teóricas y potencialidad política de estas perspectivas en la situación histórica que estamos vi-

Palabras Clave: Interseccionalidad; covid-19; precariedad de la vida; feminismos 


\section{Abstract}

This article examines how intersectional perspectives can contribute to a deeper

Keywords:

Intersectionality, Covid-19,

precariousness of

life, feminisms

\section{Introducción}

El objetivo de este artículo es analizar de qué manera las perspectivas interseccionales pueden contribuir a comprender las operaciones del poder en un contexto de crisis sanitaria y entregar a la vez una reflexión sobre las premisas teóricas y potencialidad política de estas perspectivas en la situación histórica que estamos viviendo. La evidencia empírica muestra que la pandemia es un fenómeno global que golpea en forma diferenciada a la población profundizando brechas ya existentes entre países, regiones y grupos sociales (Comité de Oxford de Ayuda contra el Hambre, Oxfam, 2021). El argumento central de este artículo es que los enfoques interseccionales no solamente nos ayudan a entender el impacto diferenciador de la pandemia, sino que también nos permiten identificar de qué forma(s) el orden social puede ser transgredido, resistido y negociado en una situación de crisis. Desde este horizonte, el artículo busca contribuir a una reflexión sobre el desarrollo teórico y conceptual de las perspectivas interseccionales en un momento crítico de transformaciones sociales en el Chile post-revuelta del $18 / 10$.

El artículo está estructurado de la siguiente manera. En una primera sección se analizan las genealogías de los feminismos interseccionales en el norte global poniendo énfasis en las condiciones geopolíticas que han (im)posibilitado la generación de espacios de saberes transformadores. En la siguiente sección se interroga de qué manera las perspectivas interseccionales nos permiten abordar tanto la profundización de desigualdades como las transformaciones del orden social que emergen en un contexto de crisis social y sanitaria. Un aspecto central es el desarrollo de una mirada que más allá del reconocimiento de subjetividades diferenciadas intenta también formular un análisis de 
los dispositivos de poder que construyen y normalizan esas subjetividades como desiguales. En una tercera sección el artículo incorpora una reflexión sobre el impacto teórico de las propuestas formuladas desde las movilizaciones feministas y su importancia para el desarrollo de las perspectivas interseccionales en un contexto de pandemia.

Antes de continuar, es necesario dejar en claro que al escribir desde una posición en la diáspora académica en Suecia, mi análisis no escapa de la condición de parcialidad inherente a la producción y distribución globalizada del conocimiento. Estos apuntes están también marcados por el compromiso político que me llevó al exilio y que atraviesa mi relación con los feminismos del sur. Esta posición de contrapunto, para usar las palabras de Edward Said (2000, p. 140), implica no solo desarraigo, sino que también algunas ventajas. Por ejemplo, la posibilidad de percibir la simultaneidad de las experiencias de la vida cotidiana en diferentes contextos culturales, así como la distancia frente a normas establecidas y sentidos comunes establecidos. Honrar esa definición es por supuesto un desafío asumido con humildad en este texto, entendiendo también que las limitaciones epistemológicas pueden contribuir al diálogo y a la búsqueda de miradas comunes.

\section{Las genealogías de los feminismos interseccionales}

Así como los procesos dia spóricos generan nuevos espacios de conocimiento, las trayectorias seguidas por conceptos y perspectivas teóricas están también permeadas por los contextos donde son recibidos y asumidos como nuevas formas de interpretar la realidad. La genealogía de la interseccionalidad aparece íntimamente ligada a los debates de emancipación femenina y a las estrategias políticas desarrolladas a partir de la década del 1970 en USA. Al mismo tiempo, la recepción de la interseccionalidad en la academia y su instalación en el canon feminista ha seguido también la lógica de divulgación del conocimiento implantada por la intensificación, a nivel global, de prácticas neoliberales en las universidades. Prácticas que, como es sabido, sostienen la hegemonía del norte global respecto a la divulgación, el reconocimiento y la valoración de saberes y sujetos de conocimiento funcionales al orden económico mundial.

La rápida circulación de los enfoques interseccionales durante las últimas décadas sugiere la relevancia de una perspectiva teórica que responda a problemáticas fundamentales en distintos espacios de producción de conocimiento. Sin embargo, hay que tener presente que se necesitó más de una década para que la noción de interseccionalidad se instalara en los espacios académicos del Norte global para seguir desde allí un recorrido 
que todavía no ha terminado. Hasta los inicios del nuevo milenio, el aporte de la jurista afroamericana Kimberlé Crenshaw (1989; 1991), estaba fundamentalmente ligado a una historiografía feminista que localizaba la llamada "tercera ola" en la persistencia de la herencia racista de la esclavitud en los Estados Unidos. En las obras de Crenshaw, la interseccionalidad es concebida como una metáfora para visibilizar la posición de las mujeres afroamericanas, a partir de formas específicas de opresión, generadas a partir de la operación simultánea de diferentes sistemas de subordinación. A juicio de la autora, tanto los discursos feministas como los antirracistas habían hasta ese momento ignorado sistemáticamente las maneras en que el patriarcado, el capitalismo y el racismo operan simultáneamente en situaciones de discriminación y violencia contra las mujeres. A partir de esta mirada, Crenshaw también expresa una crítica al movimiento feminista, que al marginalizar las experiencias de las mujeres afroamericanas, renuncia a problematizar de qué manera la subordinación de las mujeres está marcada por el racismo y la pertenencia de clase (Crenshaw, 1991).

Si bien es cierto que la contribución de Crenshaw ha sido ampliamente celebrada, la articulación entre política y teoría que ofrece la autora no siempre ha sido considerada por las y los seguidores del concepto. El desconocimiento de la tradición teórica afroamericana, de la cual se nutre el movimiento feminista antirracista, ha sido también un punto crítico en la recepción del concepto en Europa. Como señalan las teóricas feministas Avtar Brah y Ann Phoenix (2004) la construcción de la subjetividad femenina ha sido históricamente materia de disputa. Las autoras nos recuerdan el testimonio de la luchadora anti-abolicionista Sojourner Thruth quien, a partir de la experiencia de haber sufrido la esclavitud, interpeló a las participantes de la Convención por los Derechos de la Mujer en Akron, Ohio, en 1851 con la pregunta “¿No soy yo acaso una mujer?” (Brah $\&$ Phoenix, 2004, p. 76). Las palabras de Thruth cuestionan que el sufragio fuera considerado un derecho prioritario para las mujeres en circunstancias de que muchas de ellas eran sometidas a la esclavitud. Esta crítica es una clara ilustración de la imposibilidad de asumir una lucha contra el orden patriarcal sin tomar en cuenta otras estructuras de opresión. A pesar de su invisibilidad en la historiografía feminista, el discurso de Thruth recuerda que el cuestionamiento a la universalización de la condición femenina ha estado presente históricamente en las movilizaciones por los derechos de las mujeres.

Mientras la idea de la interseccionalidad es formulada en un contexto de movilización del feminismo antirracista, concentrándose en temas de poder, violencia, desigualdad y discriminación en Estados Unidos (bell hooks, 1984; Davis, 1981; Hill Collins \& Bilge, 
2016), la recepción de esta perspectiva en los países europeos ha estado atravesada por el temor a la fragmentación de lo femenino (Bilge, 2013). La ausencia de una perspectiva de poder ha sido particularmente problemática en países como Suecia que a pesar de ser ampliamente reconocido por ser un estado de bienestar promotor de los derechos de las mujeres muestra claras tendencia a la exclusión racializada y de clase (de los Reyes \& Mulinari, 2005). En este contexto, la conceptualización de las diferencias entre las mujeres se da a partir de nociones identitarias (mujeres migrantes) o normativas (grupos LGTB) que, aunque dan cuenta de las distintas subjetividades contenidas en la feminidad, no problematizan las lógicas de explotación ligadas a relaciones de dominación de clase, raza o sexualidad.

Al conceptualizar la desigualdad como un problema de paridad, el feminismo sueco ha subordinado la explotación de clase, los procesos de racialización y la imposición de la sexualidad binaria a las metas de la paridad de género. Es así que las demandas de servicio doméstico subvencionadas son formuladas a partir de una argumentación paritaria, que obviando la explotación racializada de lxs trabajadorxs de casa particular, plantea la necesidad de las mujeres suecas de hacer carrera profesional sin tener que preocuparse de labores del hogar (de los Reyes, 2016). Tradicionalmente, el modelo de bienestar sueco ha definido las tareas de cuidados dentro de las políticas públicas, beneficiando particularmente a madres jefas de hogar que trabajan o estudian. Pero, al mismo tiempo que la mercantilización de las labores reproductivas intensifican su consumo en familias de altos ingresos, las metas de paridad de género son desplazadas desde las políticas públicas del estado de bienestar a la esfera del mercado. La privatización del consumo de cuidados permite de esta manera mantener un modelo heteropatriarcal, donde los ingresos de los hogares son destinados a solucionar, en forma diferenciada, los conflictos entre trabajo remunerado y demandas domésticas.

La circulación global de las perspectivas interseccionales han abierto una discusión urgente sobre cómo conceptualizar las desigualdades en las experiencias de vida de las mujeres, así como también acerca de la interrelación de diferentes formas de subordinación. Sin embargo, los debates dentro del feminismo en los países europeos han tendido a concentrarse en identificar y jerarquizar las relaciones de poder que constituirían los núcleos centrales de la interseccionalidad, definiendo a la vez cuáles podrían ser considerados periféricos o marginales (Lewis, 2013). En este contexto, la marginalización del racismo ha sido una fuente de crecientes disputas dentro del feminismo europeo. Como señala Barbara Tomlinsson (2013), mientras no se reconozca la hegemonía de las mujeres blancas, hetrosexuales y de clase media en los discursos feministas, se se- 
guirá particularizando la tradición teórica del feminismo afroamericano y antiracista. Al mismo tiempo, indica la autora, las perspectivas interseccionales corren riesgo de ser despolitizadas y despojadas de su potencial transformador. Tomlinson entrega importantes antecedentes para analizar de qué manera la interseccionalidad es coptada por perspectivas que reconocen la diversidad de posiciones que habitan el feminismo, pero no indagan en sus causas. En este contexto, la ausencia de la teorización del impacto global del proyecto colonial, está también asociada al silencio sobre el racismo que durante mucho tiempo ha caracterizado los debates feministas en Europa.

La particularizacion del racismo, como una forma de opresión cuyos antecedentes históricos se asocian fundamentalmente a la herencia de la esclavitud en los Estados Unidos, ha sido también fuertemente cuestionada a partir de las experiencias de las diaspóras del Sur global a las metrópolisis del Norte global. La elaboración de una lectura interseccional de los patrones de desigualdad a partir de la dominación de clase, raza, género y sexualidad, desplegada al amparo de los estados de bienestar europeos, ha tenido un correlato en la idea de la colonización como un fenómeno que se reproduce en los circuitos globales de producción del conocimiento (Bilge, 2013; Brah \& Phoenix, 2004; Lewis, 2013). El aporte del feminismo postcolonial, al problematizar las maneras a partir de las cuales los migrantes de los países del sur global son incorporados como sujetos racializados en los mercados de trabajo, sistemas de bienestar y modelos de ciudadanía en las metrópolis del capitalismo global, han generado importantes elaboraciónes téoricas sobre el nexo entre colonialismo, migración y racismo global. El llamado de los feminismos decoloniales a (de)esencializar las categorias impuestas al partir del proyecto colonial (Lugones, 2012), está también reflejado en investigaciones que problematizan la (re)produccion de esas categorias a partir de las transformaciones de los patrones de acumulacion global del capital (Bhattacharyya, 2018; de los Reyes, 2016; Cavallero \& Gago, 2021; Mezzadra, 2020). Es así que la reproducción intergeneracional de subalternidades racializadas aparece como un rasgo fundamental en procesos donde la (im)posibilidad de acceder a los recursos materiales y simbólicos de los centros de poder en países de altos ingresos está reflejada en discursos nacionalistas, prácticas discriminatorias y racismo institucional (de los Reyes \& Mulinari, 2020; Erel et al., 2017; Lewis, 2013;). Al mismo tiempo, los imaginarios de familias heteropatriarcales y los vínculos biológicos han pasado a constituir también la norma en procesos cada vez más restrictivos de reunificación familiar y reconocimiento los derechos sociales de los migrantes (Sagen \& Mulinari, 2018).

Haciendo un balance del impacto de la interseccionalidad en la academia, las teóricas fe- 
ministas Carvado, Crenshaw, Reys \& Tomlinsson (2013) hacen un llamado a detenerse en las trayectorias del concepto, para evaluar de qué manera es recibido, interpretado y aplicado en diferentes contextos polititicos y académicos. Esas trayectorias indican, según las autoras, que la interseccionalidad no está enclaustrada en sus postulados iniciales ni se agota en sus múltiples interpretaciones. Más bien se podría decir que es un work in progress (proyecto en desarrollo), donde los desafíos locales también implican la adaptación y reorientación de algunas de sus propuestas originales. Desde este punto de vista, los enfoques interseccionales están abiertos a problematizar nuevas relaciones de poder y a traspasar tradiciones disciplinarias. Las autoras abren de esta manera la posibilidad de un diálogo, en el cual los desafíos asociados a condiciones históricas específicas son percibidos como una oportunidad de contribuir a generar propuestas transformadoras.

En esta línea de análisis, los conflictos planteados por lo que Jennifer Nash (2018) define como "las guerras interseccionales" tienden a concentrarse en miradas correctivas sobre la definición, origen y propiedad intelectual del concepto, olvidando preguntas centrales respecto al significado de la institucionalización de la interseccionalidad y la posibilidad de generar nuevas interrogantes. Nash sugiere volver la mirada hacia temáticas que transciendan las relaciones de propiedad y dominio, que florecen en ámbitos académicos, abriendo la imaginación en torno a vínculos de afecto, cuidado, intimidad, vulnerabilidad. Su propuesta adquiere especial urgencia en la situación actual, donde la precariedad de la vida - para usar el concepto acuñado por los movimientos feministas en Latinoamerica - se agudiza no solamente debido a las características estructurales del capitalismo neoliberal, sino que también por la situación generada a partir de las formas en que la crisis sanitaria es enfrentada a nivel nacional y global.

Una revisión de las trayectorias de la interseccionalidad durante los últimos años expone los desafíos de una perspectiva que analiza la producción de desigualdad a partir de la operación simultánea de relaciones de clase, género, sexualidad y raza e invita a reflexionar sobre las lógicas que construyen identidades esencializadas a partir de esas relaciones. Esos debates muestran también la multiplicidad de las miradas interseccionales y su adaptación a diversos desafíos teóricos, conceptuales y empíricos a nivel global. La existencia de barreras institucionales y disciplinarias han estado reflejadas en interpretaciones que buscan incorporar la interseccionalidad a una narrativa de progreso feminista - expresada entre otras cosas en la idea de las diferentes olas feministas -, desconociendo el legado teórico y político del feminismo afroamericano antiracista y el aporte teórico desarrollado a partir de las movilizaciones feministas en el Sur global. Cuando los movimientos feministas en Chile y Latinoamérica se articulan en torno a 
la formulación de estrategias transversales para superar una situación de crisis social, económica y sanitaria se abren también nuevos caminos para revitalizar el contenido transformador de las perspectivas interseccionales.

\section{Una mirada interseccional del Covid 19}

La pandemia representa una situación históricamente excepcional en la cual se combina la inseguridad sanitaria con la desestabilización económica, institucional y política a nivel global. El confinamiento ha significado un cambio radical en la vida cotidiana de las personas, a la vez que las amenazas a la salud y el aumento de la inestabilidad económica y laboral conllevan altos niveles de estrés para la gran mayoría de los hogares. Sin embargo, sabemos que los niveles de crisis e inseguridad afectan a las sociedades de manera diferenciada. Los efectos de la pandemia en el Sur Global han sido particularmente severos para las comunidades LGBTQ, minorías étnicas, migrantes, trabajadorxs de casa particular y trabajadorxs sexuales (El-Ali, 2020). Las políticas sociales relacionan esas brechas a la existencia de los llamados "grupos vulnerables", los cuales, en la gran mayoría de los casos, están directamente ligados a la desigualdad estructural de las sociedades capitalistas y a las condiciones de (des)protección generadas a partir de las prioridades establecidas por los gobiernos. Así, el informe de Oxfam, define al Covid como "El virus de la desigualdad" y señala que:

La pandemia ha afectado mucho más a quienes viven en la pobreza que a los ricos y ha tenido impactos particularmente severos en las mujeres, personas negras ${ }^{2}$, afrodescendientes, pueblos Indígenas e históricamente marginados y comunidades oprimidas en todo el mundo. Las mujeres, y sobre todo las mujeres racializadas, arriesgan perder sus trabajos debido al coronavirus en mayor medida que los hombres. En América Latina, los afrodescendientes y pueblos indígenas, ya marginados, se han visto más afectados que el resto de la sociedad; tienen más probabilidades de morir y sufrir condiciones de indigencia. (Oxfam, 2021, p. 14, traducción de la autora)

En el análisis de Oxfam, los afectados por la crisis son identificados a partir de su posición en un orden social desigual, originado en formas históricas de explotación y subordinación. La vulnerabilidad frente al Covid es aquí asociada a cuerpxs o comunidades definidos a partir de su situación de asalariadxs, racializadxs o sexualizadxs. Siguiendo el análisis desarrollando anteriormente, cabe preguntarse si las potencialidades de la interseccionalidad como instrumento analítico se agotan al visibilizar los cuerpos que habitan las posiciones más vulnerables en la sociedad y al confirmar la simultaneidad de los mecanismos de explotación y subordinación social. Al no discutir

\footnotetext{
2 Esta es una traducción textual del término en inglés "black people", que refleja una tradición política e identitaria específica y que no tiene una traducción directa al castellano.
} 
las lógicas de poder que construyen esas posiciones se corre el riesgo de esencializar a las subjetividades que las habitan. Por tanto, es necesario recordar que el potencial de la interseccionalidad está dado no solo en identificar la multiplicidad de relaciones a partir de las cuales se ejerce el poder, sino que también en interrogar la(s) lógica(s) que operan a través de la construcción de la diferencia, sobre todo en una situación de crisis que pone en juego el orden social y la distribución de recursos en la sociedad.

Al referirse a las mujeres asalariadas como racializadas, Oxfam da cuenta de las condiciones de vulnerabilidad generadas a partir de la intersección de relaciones de opresión capitalista, patriarcal y racista. Mientras que el orden capitalista aparece como un elemento central en el análisis de las condiciones imperantes en regímenes laborales diferenciados, la división entre el trabajo asalariado y el trabajo doméstico adquiere un carácter crítico en un contexto de pandemia. El impacto de la crisis sanitaria en la economía ha obligado a nuevas formas de organizar los espacios de vida, alterando la división entre trabajo productivo y trabajo doméstico, estructurado a partir de la separación entre los espacios privados y públicos, debido a las demandas del trabajo a distancia. El confinamiento obliga a la multiplicación e intensificación del uso de los espacios domésticos al mismo tiempo que las necesidades básicas de alimentación, cuidado, higiene y aseo requieren el acceso a recursos monetarios externos. En Chile, al igual que en otros países, la proliferación de ollas comunas en los sectores empobrecidos son una muestra no solo de la vulnerabilidad alimentaria producto de la crisis, sino también de la extrema fragilidad de los modelos de subsistencia (Silva, et al., 2020).

Siguiendo el llamado de la geógrafa feminista Doreen Massey (1999) a espacializar la teoría social, y entendiendo el espacio como un dispositivo ordenador de cuerpos y territorios, de los Reyes y Mulinari (2005) incorporan la dimensión espacial a la interseccionalidad, invitando a examinar las relaciones de poder que construyen espacios diferenciados como expresión de diversidad. Según se plantea, la construcción de espacios segregados permite entender no solamente el ejercicio del poder que establece barreras, sino también su normalización, al vincular la existencia de cuerpos jerárquicamente diferenciados a espacios segregados. Es así que históricamente la construcción de la división genérica - sexual ha estado íntimamente vinculada a la separación entre espacios públicos y privados. El confinamiento, al romper la dicotomía entre el mundo del trabajo y el mundo de los cuidados, abre espacios de negociación, transgresión o represión que reconfiguran el orden social establecido. Desde esta perspectiva, la intensificación de la violencia contra mujeres y niñxs no puede ser considerado un elemento circunstancial, sino más bien un componente latente de las relaciones de poder patriar- 
cal en los hogares. En esta línea, la proliferación de fronteras, entre países, regiones y cuerpos estaría expresando la fragmentación necesaria para perpetuar los regímenes de acumulación y explotación que operan a diferentes niveles del orden social (Bhattacharyya, 2018; Mezzadra, 2018).

Al mismo tiempo que las personas enfrentan la pandemia en espacios diferenciados, los recursos asociados a esos espacios generan nuevas desigualdades en el acceso al cuidado y al consumo. Los espacios comunes de esparcimiento (parques, plazas y bibliotecas) han sido reducidos a lugares mercantilizados (centros comerciales), a la vez que nuevas y viejas formas de consumo demandan la circulación de cuerpos expuestos a los embates de la pandemia y a la explotación laboral. Este es el caso, por ejemplo, de lxs trabajadorxs de casa particular o de las personas ocupadas en las plataformas de distribución de alimentos. Si bien es cierto que la lógica de explotación capitalista es central para entender la agudización de las formas de dominación que emergen en una situación de pandemia, la espacialidad nos permite identificar algunas de las particularidades de las operaciones de poder, generadas a partir de la separación, confinamiento y circulación de cuerpos diferenciados. En este contexto, el rol del Estado aparece como un elemento fundamental en el análisis de los efectos de la pandemia en cuerpos diversos.

Las políticas estatales no solo tienen un impacto directo en la seguridad sanitaria, sino también en las condiciones de supervivencia de los hogares. Al intentar mantener el balance entre la economía y la seguridad sanitaria los estados han profundizado las brechas existentes. En un informe de la Organización de las Naciones Unidas (ONU, 2020), se indica que los esfuerzos estatales no han sido suficientes para contrarrestar los efectos del Covid en mujeres, niñxs y personas pertenecientes a comunidades LGTBTIQ+. La crítica de Oxfam (2021), más que apuntar a deficiencias coyunturales, denuncia el impacto negativo de las últimas décadas de políticas de austeridad en la capacidad de los servicios públicos para enfrentar la crisis. La ausencia de políticas públicas destinadas a contrarrestar los efectos de la pandemia no es casual, sino que corresponden a un diseño político en el cual el derecho a la salud no es reconocido como tal. Sin embargo, más allá de las políticas macroestructurales del estado neoliberal, que favorece la mercantilización de la seguridad sanitaria, hay evidencias que indican que percepciones normativas de género, clase y sexualidad también influyen en las condiciones de acceso a servicios de salud a nivel micro. El trato discriminatorio a personas en situación de calle, minorías étnicas y comunidades LGTBIQ+ en los servicios de salud es una muestra más de la desigualdad de acceso a la seguridad sanitaria (El-Ali, 2020). 
Las prácticas discriminatorias en la atención sanitaria se enmarcan, por tanto, en una gestión política que, al favorecer el funcionamiento del mercado y la continuidad de la acumulación del capital, evidencia lo que el filósofo camerunés Achille Mbembe (2003) ha llamado necropolítica. Mbembe sitúa los orígenes de la necropolítica en el proyecto colonial y en la larga historia de violencia, despojo y genocidio que acompañó la expansión mercantil europea. La persistencia de este fenómeno en la sociedad actual, señala Mbembe, está ligada fundamentalmente al funcionamiento del poder en el capitalismo globalizado de hoy. La necropolítica es interpretada por algunos como la cara oculta de lo que el filósofo frances Michael Foucault llamó biopolítica, es decir, el poder soberano de controlar cuerpos y regular el derecho a la vida y la muerte (Foucault 2014). Al hablar de necropolítica, Mbembe va más allá. El dejar morir, más que el simple ejercicio del poder, es también constitutivo de un orden global donde habitan tanto la (ir)racionalidad de la política, así como las prácticas cotidianas que construyen otras formas de vida como subordinadas, prescindibles y a la larga también sacrificables a intereses superiores.

En Chile, al igual que en otros territorios colonizados, la necropolítica ha operado no solo a través del aniquilamiento de cuerpos, comunidades y cosmologías subalternas, sino también mediante el despojo sistemático y masivo de las condiciones materiales que sostienen la existencia de formas de vida prescindibles a la acumulación capitalista. En un contexto de pandemia, la priorización de las actividades productivo-mercantiles ligadas a la acumulación de capital no es casualidad. Tampoco lo es la actuación del Estado, que transfiere recursos comunes a las grandes empresas a través de licitaciones, excepciones tributarias y privatización de bienes naturales. La política del dejar morir se expresa tanto en la profundización de los mecanismos de explotación como en la falta de resguardo de las condiciones de supervivencia de los hogares. En estos momentos de pandemia presenciamos la de-territorialización de las zonas de sacrificio y su instalación en espacios urbanos donde un porcentaje creciente de trabajadorxs buscan el sustento cotidiano, a pesar del riesgo de contagio. El aumento del desempleo, las bajas pensiones y el creciente endeudamiento tensionan aún más condiciones de vida estructuralmente precarias. La violencia estructural del orden social está reflejada no solamente en el aumento de la represión estatal a las protestas ciudadanas, sino también en el maltrato a mujeres y niñxs en el ámbito familiar.

En la crisis actual, donde la precariedad del presente se combina con la (im)posibilidad de futuros viables para las grandes mayorías, es necesario detenerse en la continuidad 
histórica entre el proyecto colonial y las modalidades actuales de acumulación del capital, para entender de qué manera la construcción de territorios y cuerpos diferenciados está asociada a los parámetros de desigualdad global que se han agudizado en un contexto de pandemia. Así como la proliferación de fronteras nos permite develar los efectos diferenciadores del capitalismo global (Bhattacharryya, 2018; Mezzadra, 2020), las perspectivas interseccionales abren la posibilidad de articular conceptualmente la fragmentación de espacios con la diferenciación de cuerpos y territorios. De esta manera, es posible también avanzar en la comprensión de las lógicas de poder que construyen la precariedad de la vida, como un elemento transversal del orden social. Sin embargo, no hay que olvidar que la transversalidad de la precarierad de la vida es también lo que hoy hace posible la articulación de resistencias y el surgimiento de movilizaciones interseccionales en torno a la valorización de la economía de los cuidados, la defensa de la vida y los bienes comunes, el respeto por la soberanía de los cuerpos y la recuperación de memorias que permitan ir tejiendo otros futuros.

\section{Resistencias interseccionales}

Las contribuciones de las perspectivas interseccionales al análisis de la pandemia aparecen aún más necesarias en un contexto de fragmentación social exacerbada tras décadas de neoliberalismo. El uso del lenguaje de la interseccionalidad en los movimientos feministas latinoamericanos permite no solamente identificar las divisiones que construyen las múltiples prácticas del poder, sino que también cuestionarlas y proponer alternativas que puedan transgredir el orden establecido a través de esas divisiones. Los aportes feministas al análisis de la violencia como fenómeno sistémico y transversal son aquí fundamentales. Las perspectivas interseccionales permiten entender los nexos que articulan el ejercicio de la violencia a nivel individual, institucional y estructural, a la vez que visibilizan de qué manera la violencia actúa sobre cuerpos diversos (Oyarzún Vaccaro, 2018; Troncoso y Follegatti, 2019). Un ejemplo de esta articulación es la muy celebrada performance del grupo Las Tesis "Un violador en tu camino", que fuera replicada en múltiples lugares del mundo convocando a mujeres de diferentes edades, nacionalidades y orientación sexual. La fuerza de la interpelación de Las Tesis puede ser entendida tanto a partir del momento específico de represión estatal en Chile y las denuncias de violencia sexualizada hacia lxs manifestantes, pero también en el marco del violento disciplinamiento que históricamente ha construido la hegemonía de cuerpos binarios. La de-domesticación de la violencia y la denuncia de su carácter sistémico emerge de esta manera como un sitio de resistencia desde las movilizaciones feministas. 
Como señalan las teóricas feministas Luci Cavallero y Verónica Gago (2021) al analizar el impacto del endeudamiento como ejercicio de violencia sobre cuerpos feminizados, los enfoques interseccionales permiten politizar las conexiones entre demandas aparentemente separadas y/o marginalizadas y construir un lenguaje común en torno a las transformaciones sociales. La interseccionalidad es, de acuerdo a esta perspectiva, no solamente lo que permite visibilizar posiciones jerárquicamente diferenciadas, sino también una herramienta para comprender las múltiples maneras en que el capitalismo opera sobre las vidas de las personas. El análisis feminista del endeudamiento nos lleva, según Cavallero y Gago, a interrogar la temporalidad de los dispositivos de poder y las distintas formas de resistir a ellos. Así como el endeudamiento posibilita la satisfacción de una necesidad inmediata, también conlleva el sacrificio de la autonomía a futuro. Desde una perspectiva interseccional es posible problematizar de qué manera la temporalidad influye en la normalización de la desigualdad, bajo la promesa de un futuro inalcanzable. Como el historiador Dipesh Chakrabarty (2000) ha señalado, la idea del not yet (no todavía) está profundamente enraizado en el imaginario (post)colonial, que a partir de una percepción linear de la historia, construye la desigualdad del presente como diferentes etapas de un destino universal. En este contexto, los trabajos de memoria desde una perspectiva interseccional abren también la puerta a la problematización del impacto de la(s) historia(s) recientes en la articulación de resistencias colectivas a la violencia del orden neoliberal.

La crisis sanitaria se da en Chile en el marco de una revuelta social que pone de manifiesto el carácter represivo del modelo neoliberal y la incapacidad gubernamental de implementar medidas que efectivamente puedan solucionar las necesidades de la población. Al mismo tiempo, es necesario señalar que la impugnación al orden neoliberal que explotó a partir de la revuelta está también marcada por la intensificación de las movilizaciones sociales, que a través de diferentes canales buscaban articular alternativas a este orden. El mayo feminista de 2018 y los Encuentros plurinacionales de mujeres que luchan representan, entre otras iniciativas, la creación de plataformas alternativas a la institucionalidad vigente desde lo colectivo. Las prácticas deliberativas, los modelos de representación y la formulación de agendas aglutinadoras desde los territorios son signos de resistencia a un modelo de sociedad donde el conformismo (en la medida de lo posible), la corrupción y el individualismo habían hecho de lo común un espacio de despojo (Elgueta \& Marchant, 2020). En este contexto, la rearticulación del tejido social que ya estaba en marcha antes de la pandemia conforma una plataforma de movilización que permite también enfrentar la emergencia sanitaria. Un ejemplo de esto 
es el llamado de la Coordinadora $8 \mathrm{M}$ a organizar territorialmente el apoyo a víctimas de violencia familiar, la organización de ollas comunes, reparto de alimentos y agua en lugares desprotegidos son también ejemplo de estas iniciativas comunitarias.

Las resistencias interseccionales aparecen también en el cuestionamiento de la división entre trabajos reproductivos y productivos. Esto cobra especial relevancia en un contexto de pandemia, donde la visibilidad de los trabajos de cuidado está dada no solo a partir de las necesidades sanitarias, sino también debido al confinamiento y localización de (algunas) tareas asalariadas dentro de los espacios domésticos. El rol fundamental de los trabajos de cuidado en el sector salud, aunque reconocido y celebrado, es apenas compensado en términos salariales. Tampoco se sabe de qué manera la sobrecarga laboral ha incidido en la organización del trabajo doméstico. El concepto pobreza de tiempo desarrollado por las investigadoras Andrea Sato y Francisca Barriga (2021) devela la desigualdad generada a partir de la distribución de tareas domésticas dentro del hogar y que debido a estereotipos de género afecta mayoritariamente a las mujeres. El confinamiento pone también limitaciones al acceso de trabajo doméstico asalariado, lo cual actualiza la importancia de estas tareas, que en la mayoría de los casos son invisibilizadas y devaluadas.

La incorporación de la idea de la sustentabilidad de la vida, desarrollada por la economista feminista Cristina Carrasco Bengoa (2016), es un aporte fundamental para enfrentar los desafíos de la reproducción en un contexto de pandemia. La sustentabilidad de la vida se refiere a una visión sistémica que articula la urgencia de abordar la sustentabilidad a varios niveles; desde la naturaleza, pasando por las comunidades y llegando a los hogares. A diferencia de las perspectivas de la economía ortodoxa, que considera la producción y la reproducción cómo ámbitos separados, y profundizando las alternativas levantadas por economistas feministas que postulan la interdependencia de ambas esferas, Carrasco Bengoa enfatiza la necesidad de colocar la sustentabilidad de la vida como la prioridad fundamental de la economía política. De esta manera, las voces feministas que durante los últimos años han identificado la precarización de la vida como un problema central del capitalismo contemporáneo, encuentran un eco en la elaboración de alternativas conceptuales que desde la academia dialogan con las demandas planteadas desde el movimiento feminista.

\section{Apuntando al futuro}

¿Qué se puede decir de la relevancia de las perspectivas interseccionales en un contexto 
de pandemia? ¿Qué formas de resistencia se generan en este contexto? Estos apuntes dan cuenta de las genealogías del concepto y su circulación en distintos ámbitos académicos, para detenerse en la relevancia de la interseccionalidad en el análisis del Chile post-pandemia. Siguiendo las huellas de esas trayectorias y atendiendo a la necesidad de superar la fijación categorial que ha caracterizado gran parte de los debates académicos en los países del Norte global, es posible concluir que el reconocimiento del carácter contingente de la interseccionalidad es central. De esta manera, las lecturas del poder ejercido en torno a la simultaneidad de las relaciones de clase, género, sexualidad y raza, nos permiten visibilizar las subjetividades construidas a partir de las desigualdades del orden social, pero nos indican también la necesidad de interrogar sobre las lógicas constitutivas de ese orden y la forma en que se expresan y son desafiadas en contextos específicos.

Los aportes a la interseccionalidad desde los feminismos del sur están íntimamente ligados a un auge de las movilizaciones feministas. Son generadas desde un momento político en que los espacios de deliberación, formulación de demandas y organización colectiva constituyen prácticas que desbordan los contenidos tradicionales del orden político establecido. Si bien es cierto que la interseccionalidad ha contribuido a crear un lenguaje común para abordar la desigualdad que habita en cuerpos diversos, es posible constatar que las prácticas y reflexiones que surgen en las movilizaciones inscriben también nuevos significados en las bitácoras interseccionales. Así como la incorporación de la temporalidad y la espacialidad nos permiten abordar conceptualmente - y traspasar políticamente - los procesos de diferenciación a partir de los cuales operan las relaciones de poder, la identificación de la precariedad de la vida (y la defensa de su sustentabilidad) muestran también que la interseccionalidad puede ser un instrumento eficaz para articular resistencias y proponer nuevos y mejores futuros.

\section{Referencias bibliográficas}

Barriga, F. y Sato, A. (2021). Pobreza de Tiempo y Desigualdad: La reproducción del Capital desde una mirada feminista. Fundación Sol.

hooks, b. (1984). Feminist Theory. From margin to Center. Pluto Press.

Bhattacharryya, G. (2018). Rethinking Racial Capitalism. Questions of Reproduction and Survival. Rowman \& Littlefield. 
Bilge, S. (2013). Intersectionality undone. Saving intersectionality from feminist intersectionality studies. Du Bois Review: Social Science Research on Race, 10(2), 405-424. DOI:10.10170 S1742058X13000283

Brah, A. \& Phoenix, A. (2004). Ain't I A Woman? Revisiting Intersectionality. Journal of International Women's Studies, 5(3), 75-86. https://vc.bridgew.edu/jiws/vol5/iss3/8

Carvado, D. W., Crenshaw, K. W., Mays, V. M., \& Tomlinson, B. (2013). Intersectionality: Mapping the Movements of a Theory. Du Bois Review: Social Science Research on Race, 10(2), 303-312. Doi: 10.1017/S1742058X13000349

Carrasco Bengoa, C. (2016). Sostenibilidad de la vida y ceguera patriarcal. Una reflexión necesaria. ATLÁNTICAS - Revista Internacional de Estudios Feministas.

https://doi.org/10.17979/arief.2016.1.1.1435

Cavallero, L. \& Gago, V. (2021). A feminist reading of debt. Pluto Press.

Collins Hill, P. \& Bilge, S. (2016). Intersectionality. Polity Press.

Crenshaw, K. (1989). Demarginalizing the Intersection of Race and Sex: A Black Feminist Critique of Antidiscrimination Doctrine. University of Chicago Legal Forum, 1989(8), 139-168.

Crenshaw, K. (1991). Mapping the Margins: Intersectionality, Identity, and Violence Against Women of Color. Stanford Law Review, 4, 1241-1300.

Chakrabarty, D. (2000). Provincializing Europe. Postcolonial Thought and Historical Difference. Princeton Studies in Culture.

Davis, A. (1981). Women, race and class. Random house.

de los Reyes, P. (2016). When feminism became gender equality and anti-racism turned into diversity management. In L. Martinsson, G. Griffin, \& K. G. Nygren (Eds.), Challenging the myth of gender equality in Sweden (pp. 23-47). Policy Press.

de los Reyes, P., \& Mulinari, D. (2005). Intersektionalitet; kritiska reflektioner över (o) jämlikhetens landskap. Liber. 
de los Reyes, P., \& Mulinari, D. (2020). Hegemonic Feminism Revisited: On the Promises of Intersectionality in Times of the Precarisation of Life. NORA - Nordic Journal of Feminist and Gender Research, 28(3), 183-196. DOI: 10.1080/08038740.2019.1705905

El-Ali, N. (2020). Covid-19 and feminism in the Global South: Challenges, initiatives and dilemmas. European Journal of Women's Studies, 27(4), 333-347.

DOI: $10.1177 / 1350506820943617$

Elgueta, G. y Marchant C. (Eds.) (2020). De la marcha y el salto. Chile Octubre 2019. Tiempo Robado Editoras.

Erel, U., Reynolds, T., \& Kaptani, E. (2017). Migrant mothers' creative interventions into racialized citizenship. Ethnic and Racial Studies, 41(1), 55-72.

DOI.org/10.1080/01419870.2017.1317825

Foucault, M. (2014). Biopolitikens födelse. Collège de France 1978-1979. Tankekraft.

Lewis, G. (2013). Unsafe Travel: Experiencing Intersectionality and Feminist Displacements', Signs: a journal of women and culture, 38(4), 869-892.

https://doi.org/10.1086/669609

Lugones, M. (2012). Interseccionalidad y feminismo decolonial. En R. Grasfoguel y R. Almanza Hernandez (eds.), Lugares decoloniales: espacios de intervención en las Americas (pp.119124). Editorial Pontificia, Universidad Javeriana.

Massey, D. (1999). Negotiating disciplinary boundaries. Current Sociology, 47(4), 5-12. DOI. org/10.1177/0011392199047004003

Mbembe, A. (2003). Necropolitics. Public Culture, 15(1),11-40. DOI.org/10.1215/0899236315-1-11

Mezzadra, S. (2018). In the Marxian Workshops. Producing subjects. Rowman \& Littelfield.

Mezzadra, S. (2020). Challenging borders. The legacy of postcolonial critique in the present conjuncture. Soft Power Revista euro-americana de teoría e historia de la política y del derecho, 7(2), 21-44. DOI.org/10.14718/So!Power.2020.7.2.2 
Mulinari, D. \& Sager, M. (2018). Safety for whom? Exploring femonationalism and care racism in Sweden. Women's Studies International Forum, 68, 149-156.

DOI.org/10.1016/j.wsif.2017.12.002

Nash, J. (2018). Black feminist reimagined. After intersectionality. Duke University Press.

Comité de Oxford de Ayuda contra el Hambre. (2021). The inequality virus. Oxfam International.

Organización de las Naciones Unidas. (2020). The impact of Covid -19 on women. www.un.org

Oyarzún Vaccaro, K. (2018). Feminismos chilenos. Una democratización encarnada. Revista Anales, 14(7), 31-50. DOI: 10.5354/0717-8883.2018.51134

Said, E. (2000). Från Exilen. Essäer 1976-2000. Ordfront.

Silva, R., de los Reyes, P., y Galaz, C. (2020). Policy brief. Cuerpos diversos e inclusión (in) subordinada. Una propuesta de políticas urbanas interseccionales.

https://doi.org/10.34720/vkvm-3f34

Tomlinson, B. (2013). To tell the truth and not get trapped: Desire, distance, and intersectionality at the scene of argument. Signs, 38(4), 993-1018.

DOI.org/10.1086/669571

Troncoso, L., Follegatti, L. y Stutzin, V. (2019). Más allá de una educación no sexista: aportes de pedagogías feministas interseccionales. Pensamiento Educativo. Revista de Investigación Educacional Latinoamericana, 56(1), 1-15.

DOI:10.7764/PEL.56.1.2019.1

\section{Agradecimientos}

Proyecto de Investigación N²01605144, "Migrant mothers - Racialized children. Dilemmas, struggles and visions", financiado por el Consejo de Investigación sueco -The Swedish Research Council (Vetenskapsrådet dnr 2016-05144). El trabajo presentado en este artículo ha sido posible gracias a una estadía de investigación en Chile, financiada por el Consejo de investigación Sueco y por la Facultad de Ciencias Sociales de la Universidad de Estocolmo. La autora agradece también el auspicio de la Facultad de Ciencias Sociales (FACSO) de la Universidad de Chile. 


\section{Biografía de la autora}

Paulina de los Reyes obtuvo su doctorado en la Universidad de Uppsala y es actualmente Profesora Titular de Historia Económica en la Universidad de Estocolmo. Ha dirigido numerosos proyectos de investigación y participado en diversas redes académicas donde ha desarrollado su interés por las teorías interseccionales, el feminismo postcolonial, la economía feminista y las transformaciones del capitalismo contemporáneo. También ha sido presidenta de la Asociación de Investigadoras en Género de Suecia (Genus Forskarförbundet) y participó en el proyecto internacional Mujeres rumbo a Gaza (2016). ORCID ID: https://orcid.org/0000-0002-3824-4698 